\title{
Neural antecedents of self-initiated actions in secondary motor cortex
}

\author{
Masayoshi Murakami, M Inês Vicente, Gil M Costa \& Zachary F Mainen
}

The neural origins of spontaneous or self-initiated actions are not well understood and their interpretation is controversial. To address these issues, we used a task in which rats decide when to abort waiting for a delayed tone. We recorded neurons in the secondary motor cortex (M2) and interpreted our findings in light of an integration-to-bound decision model. A first population of M2 neurons ramped to a constant threshold at rates proportional to waiting time, strongly resembling integrator output. A second population, which we propose provide input to the integrator, fired in sequences and showed trial-to-trial rate fluctuations correlated with waiting times. An integration model fit to these data also quantitatively predicted the observed inter-neuronal correlations. Together, these results reinforce the generality of the integration-to-bound model of decision-making. These models identify the initial intention to act as the moment of threshold crossing while explaining how antecedent subthreshold neural activity can influence an action without implying a decision.

Decision-making involves the selection of goals or actions, but it also requires determination of the timing of action. When there is a stimulus or cue to react to, the problem of when to act is constrained by sensory and motor requirements and may involve balancing of speed versus accuracy and other facets of sensorimotor coordination, which have been intensively studied ${ }^{1}$. In reaction time tasks, decisions are associated with trial-to-trial variations in response time. These fluctuations have been theorized to arise from the integration of noisy sensory signals by a neural integrator, whose crossing of activation threshold triggers an action ${ }^{2-4}$. In support of this theory, neurons in the primate frontal cortex, parietal cortex and superior colliculus show gradual increases in neural activity after presentation of visual stimuli, reaching a constant activity threshold at which a saccadic eye movement is generated; reaction times are correlated with rates of such ramping activity ${ }^{5-7}$.

In the absence of immediate sensory stimuli, the timing of more 'spontaneous' actions is also important. Action initiation may be affected by internal state (for example, urgency signals) but, as with reaction times, contains variability. An important instance of spontaneous action generation is deciding when to give up waiting for an anticipated event whose occurrence or timing is uncertain. This form of inter-temporal choice ${ }^{8}$ - between waiting and giving up-is relevant in the context of impulsivity. Humans often choose to wait for future events, but subsequently succumb to an immediately available option while waiting ${ }^{9,10}$. Some of the neural substrates of choices between immediate and delayed rewards have been identified ${ }^{11,12}$, but comparatively little is known about the timing of decisions to terminate waiting during a delay.

Insight into spontaneous actions comes from studies of volitional or self-initiated actions, which have used what are essentially waiting tasks with low time pressure. Classical studies identified evoked readiness potentials in the midline higher motor, parietal and prefrontal cortices that precede actions, with a slow building potential even preceding the subject's reported intention to move ${ }^{13,14}$. Single units have been recorded during such tasks, revealing slow ramping activity peaking around the time of action initiation ${ }^{15-18}$.

Together, these studies are consistent with the hypothesis that an integration-to-bound mechanism, commonly used to explain perceptual decisions and reaction times, might also be a mechanism for spontaneous action timing. We sought to substantiate this hypothesis by providing more rigorous evidence for the existence of a population of neurons displaying ramping activity resembling the decision variables or integrated evidence observed in the lateral intraparietal area. A further critical issue in the case of spontaneous decisions is the origin of the signals that drive the input integrator when there is no sensory cue or evidence to accumulate. The putative input neurons would contribute causally to the determination of action timing, being partly predictive, but individual neurons would need to cooperate to reach threshold for a decision. As no such input neurons for spontaneous decisions have been described to date, identifying their properties would be important.

To test these hypotheses, we devised a waiting task in which a small reward was available immediately, but a large reward was signaled after a longer, randomized delay. Rats tested on this task frequently aborted waiting, and the timing of these aborts varied greatly across trials, with relatively minor contributions of trial history. We focused our study on the rostral secondary motor cortex (M2, the anterior region of medial agranular cortex) an area associated with action planning and spontaneous action initiation ${ }^{19-21}$, which is considered to be homologous to primate supplementary motor areas ${ }^{22,23}$. Using multi-electrode recording from M2, we first identified a population of neurons with ramping activity that met strict criteria associated with the output of putative neural integrators, but not previously described in rodents. Furthermore, we identified a second population 
of transiently active neurons whose rates fluctuated substantially from trial-to-trial in a manner that correlated with waiting time. Using a simple quantitative model, we estimated that to match the observed strength and frequency of correlation between individual transient neurons and waiting time, substantial correlations would have to exist between pairs of transient neurons. Trial-by-trial correlation analysis of simultaneously recorded pairs confirmed this prediction. Thus, a parsimonious account of our data suggests that the origin of the variable timing of spontaneous actions reflects, in part, neural transients in M2 that are integrated to produce ramping activity. The results reinforce the generality of the neural integration-to-bound theory of decision-making and identify a previously unknown population of neurons in the motor system itself participating in self-initiated actions. Furthermore, they help to clarify the interpretation of wellknown experiments conducted in human subjects concerning the neural antecedents of conscious decisions to act ${ }^{14}$.

\section{RESULTS}

\section{Behavior}

In the waiting task (Fig. 1a and Online Methods), a rat initiated waiting by inserting its snout into the waiting port. Shortly thereafter (T1 delay $=0.4 \mathrm{~s})$, the first tone (tone 1$)$ was played, after which the rat could garner a small amount of water reward at the reward port. If the rat waited for a second tone (tone 2), with a delay drawn randomly from an exponential distribution (T2 delay), it could garner a larger reward (two- to fourfold larger than the small reward). Thus, a rat waiting past tone 1 experienced an ongoing conflict between continuing to wait for tone 2 and leaving the port to collect a small reward. A value of T2 delay was chosen so that subjects performed with an $\sim 30 \%$ success rate in waiting trials (Online Methods).

Trials could be classified into three types (Fig. 1a-c). In a small fraction of trials, rats left the port before tone 1 (short-poke trials, $8.3 \pm 4.6 \%$, mean \pm s.d., $n=37$ rats). In a second class of trials, the rat responded after tone 2 (patient trials, $32.9 \pm 1.4 \%$ ). In the third class, rats left the port after tone 1, but before tone 2 (impatient trials, $58.8 \pm 3.9 \%)$. Notably, in the impatient trials, the waiting time showed substantial trial-to-trial variability, as indexed by the difference between the 90th and 10th percentiles waiting time ( $\Delta \mathrm{WT}[0.1-0.9]$ : median $=1.31 \mathrm{~s}$, range $=0.59-3.06 \mathrm{~s}$; Fig. $\mathbf{1 c}, \mathbf{d})$ and the average difference in waiting times from tone 1 of two consecutive impatient trials normalized by a sum (CV2, $0.63 \pm 0.11$; Online Methods and Fig. 1e). Median waiting time was highly correlated with $\Delta \mathrm{WT}$ [0.1-0.9] $\left(R=0.86, P<10^{-10}\right)$.

In impatient trials, rats did not usually respond promptly to tone 1 , but often stopped waiting after hundreds of milliseconds or seconds, but before tone 2 . Two observations indicate that, in impatient trials, rats intended to wait for the large reward and were not simply responding slowly to the first tone and understood the rules of the task. First, when rats succeeded in waiting for the second tone, they responded promptly $(167 \mathrm{~ms}$, median across rats, comparable to a

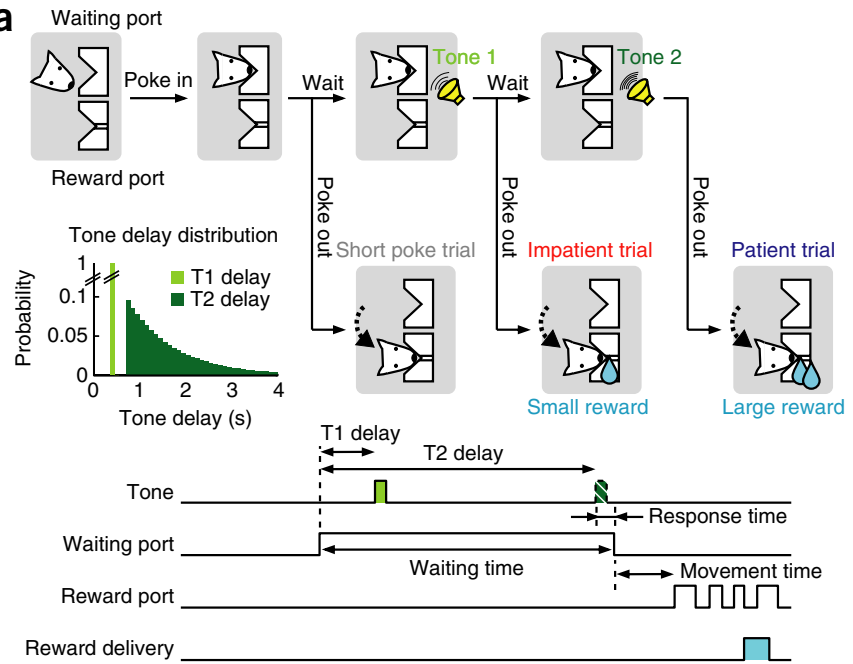

Figure 1 The waiting task and the behavioral results. (a) Schematic of trial events in the waiting task (top). In each trial, after waiting for a certain period at the waiting port, the rat received a tone(s), moved to the reward port and received a water reward, the size of which depended on the number of the tones presented. Inset, probability distributions of the delays to tone 1 ( $T 1$, light green) and tone 2 (T2, dark green). Bottom, timeline of the task events and the definition of the behavior parameters. The light green rectangle indicates the presentation of tone 1 , the dark green rectangle represents tone 2 and the light blue rectangle indicates reward. Tone 2 is represented by a hatched rectangle to indicate it was not played in the impatient trials.

(b) Snapshot of the waiting behavior. The waiting period in each trial is indicated as a gray bar. Light green ticks represent a presentation of tone 1 and dark green ticks represent tone 2. (c) Waiting time histograms of short poke trials (gray), impatient trials (red) and patient trials (blue) of an example rat. The histograms show data pooled across sessions. Inset, cumulative histogram of waiting times in impatient trials from this rat. The arrow indicates the range from 10th to 90th percentile waiting times ( $\Delta$ WT [0.1-0.9]). (d) Distribution of $\Delta W T[0.1-0.9]$ across rats. Filled bars indicate electrophysiology rats. (e) Distribution of CV2 across rats. Filled bars indicate rats used for electrophysiology. (f) A histogram of response time to tone 2 of an example rat (dark blue, $n=1,501$ trials). Light blue-shaded area indicates $95 \%$ range of response time histograms from shuffled data. The peak response time is indicated by an arrowhead. (g) Distribution of peak response time across rats. Significant peak is shown in dark blue and non-significant peak in black. Filled bars indicate rats used for electrophysiology (all were significant). b
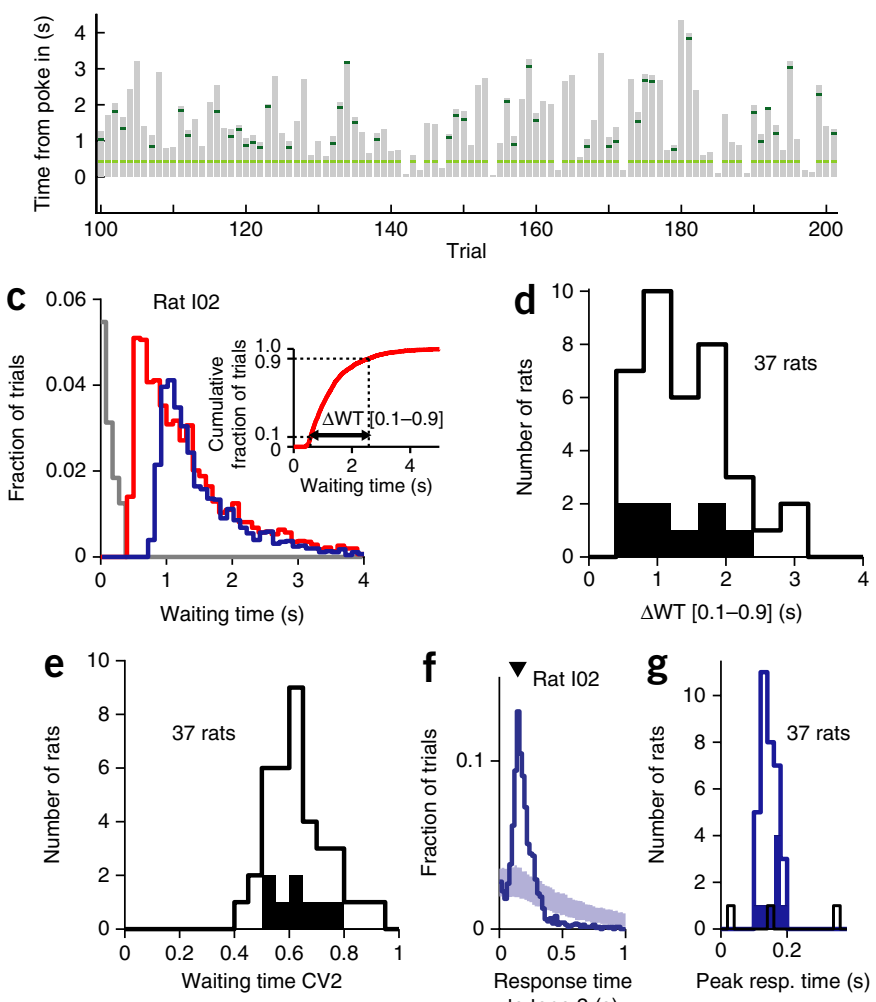
Figure 2 Difference in movement times in impatient and patient trials. (a) A scatter plot indicating median movement time in the impatient and the patient trials of different sessions of an example rat. Each gray circle indicates median movement time of the impatient trials and that of the patient trials from one session. The black circle indicates the mean of the median movement times of the impatient trials and that of the patient trials. Error bar represent \pm s.e.m. (b) Normalized mean movement times for impatient (red) and patient (blue) trials. For each rat, the movement time is normalized with movement time of the impatient trial. Error bar represents \pm s.e.m. Gray circles represent the normalized movement times of the patient trials of individual rats. Filled circles indicate rats used for electrophysiology. Movement time in patient trials was significantly faster than that in impatient trials (Wilcoxon signed-rank test, $P<0.001, n=37$ rats).
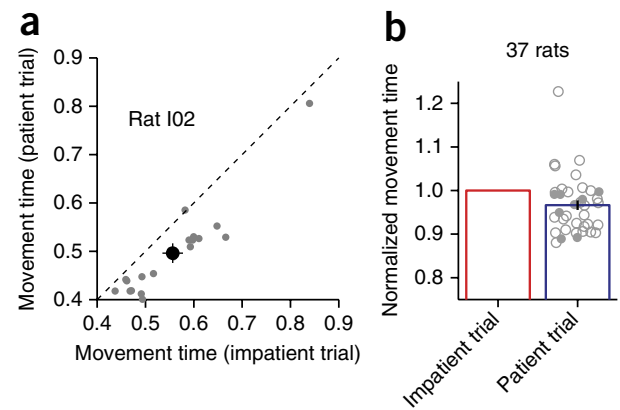

100-200 $\mathrm{ms}$ in an auditory response task ${ }^{24}$; Fig. 1f,g and Supplementary Fig. 1). Second, rats moved more quickly to the water port on patient than on impatient trials (Fig. 2), indicating that impatient and patient trials of similar waiting times were associated with different reward expectations ${ }^{25}$.

\section{Ramp-to-threshold activity in M2}

To determine specific computations performed by M2 for waiting time decisions, we next used chronic tetrode arrays to record neuronal activity while rats performed the waiting task. We recorded a total of 385 neurons in 8 rats from M2. A large fraction of neurons in M2 showed task-modulated activity in different phases of the waiting task (Supplementary Fig. 2). We mainly focused on impatient trials because, in those trials, the rats decided when to leave the port (stop waiting), independent of overt cues. The high trial-to-trial variability of waiting time in impatient trials provided a strong signal with which to correlate with neural activity.

We first searched for neurons with ramp-to-threshold activity that might serve as an internal trigger for initiating a response. We selected for neurons meeting two criteria: the firing rate reached a constant threshold before movement initiation and the timing of threshold crossing was correlated with waiting time with a regression slope close to unity (Online Methods). An example neuron meeting these criteria is shown in Figure 3. The firing rate of this neuron gradually ramped up during the waiting period (Fig. 3a), reaching the same firing rate just before the poke out (Fig. 3b). The time for the firing rate to cross a 57 spikes per s threshold, the highest threshold level we tested for this neuron, was highly correlated with the waiting time of the rat (Fig. 3c and Online Methods). The regression slope was close to unity, suggesting that the latency to the poke out from the threshold crossing time was constant.

We focused on 105 of 385 recorded neurons that showed reliable activation (48) or suppression (57) at the poke-out periods (Online Methods). Among this population, 27 neurons (20 of 48 activated neurons, 7 of 57 suppressed neurons, $7.0 \%$ of all recorded) met the criteria for a ramp-to-threshold neuron (threshold crossing time correlated with waiting time with regression slope close to unity) (Supplementary Fig. 3). By comparing the observed fraction of ramp-to-threshold neurons (7.0\%) to that obtained when randomly permuting the waiting times and neural data across trials,
Figure 3 Ramp-to-threshold type predictive activity. (a-f) Example M2 neuron with ramp-to-threshold type activity. (a) Perievent time histograms (PETHs) for an M2 neuron in different waiting time trials, aligned to poke in and smoothed with a Gaussian filter (s.d. $=50 \mathrm{~ms}$ ). Impatient trials are grouped according to the waiting time, indicated by the color scale in $\mathbf{b}$, and consistent throughout the figure. Dashed lines in PETHs indicate times at which a rat already left the port in some of the trials in that group. Threshold (57 spikes per s) is indicated by the horizontal solid line. (b) PETHs for the neuron presented in a, aligned to poke out. (c) Time to cross a threshold firing as a function of mean waiting time. The analyses with the highest and lowest thresholds with significant correlation (57 spikes per s (triangle) and 16 spikes per $\mathrm{s}$ (inverted triangle), respectively) are shown. $R=0.99, P<0.001, n=9$ for the 57 spikes per s threshold; $R=0.86, P=0.001, n=10$ for the 16 spikes per $s$ threshold. Dashed lines indicate the regression line. (d) The rate of ramping activity as a function of mean waiting time. The dashed line indicates the regression line. $R=-0.93, P<0.001, n=9$. (e) The firing
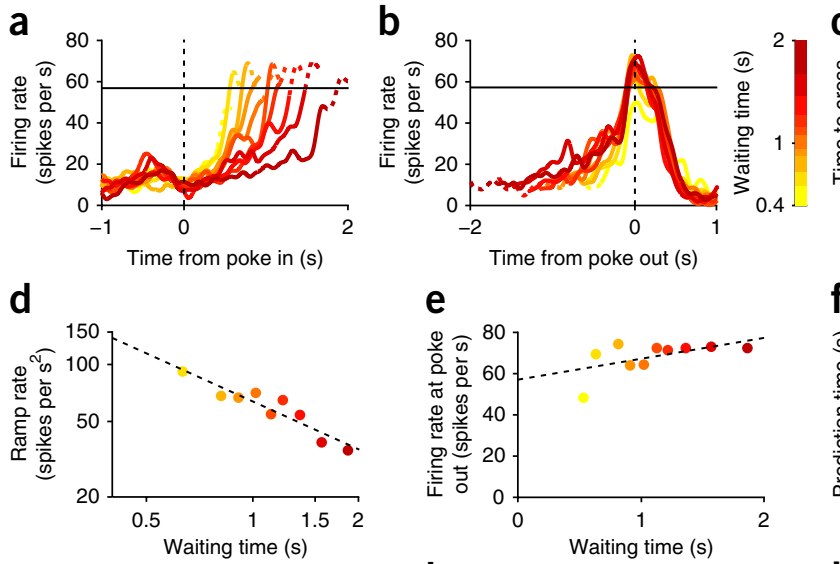

g
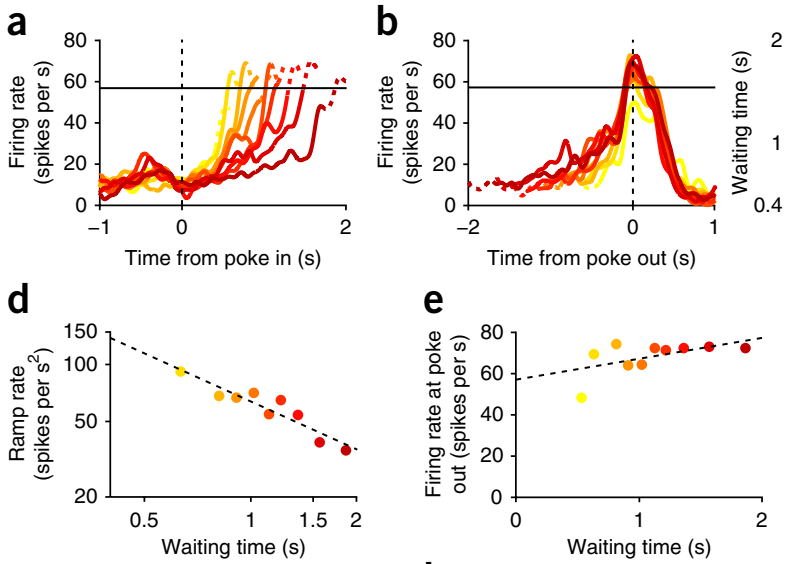

h

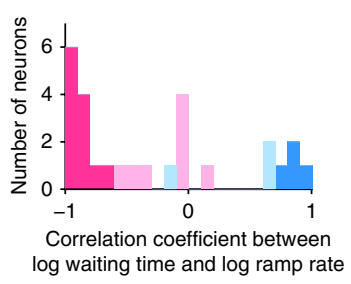

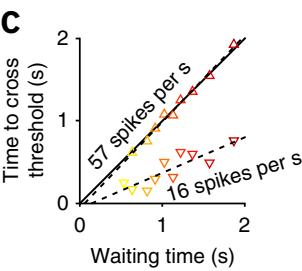

f

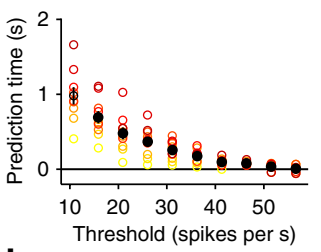

i

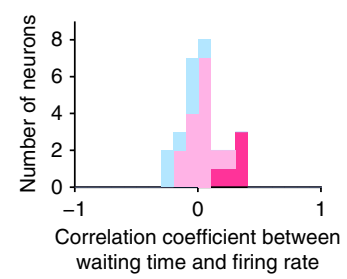

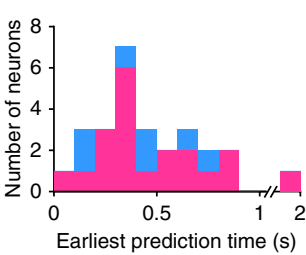

rate at the poke-out period (50-ms window before the poke out) is plotted against the mean waiting time for each group. Note that firing rate reached almost the same level at the poke out. $R=0.58, P=0.076, n=10$. (f) Difference between time to cross threshold and the waiting time (prediction time) is plotted against the threshold tested. Black circles represent mean prediction time across groups, error bars represent \pm s.e.m. and filled black circles represent significant threshold. (g-i) Population data ( $n=27$ neurons). (g) Distribution of correlation coefficients between the rate of ramping and the waiting time. Ramp-up neurons are shown in pink, ramp-down neurons in blue and neurons with significant correlation in vivid color. (h) Distribution of correlations between the firing rate at the poke-out period and the waiting time. (i) Distribution of the earliest prediction time (Online Methods). 
Figure 4 An M2 neuron with transient predictive activity. (a) Raster plots (top) represent activity of an M2 neuron, with each row corresponding to a single trial aligned to poke in (white line) and each black tick to a single spike. The impatient trials are shown on the pink background and the patient trials on the blue background. Trials are chronologically ordered from top to bottom in each type of trials. Color ticks represent tone 1 (light green), tone 2 (dark green), poke out (white) and poke in into the reward port (light blue). PETHs at the bottom represent activity in the impatient (red) and patient trials (blue), smoothed with a Gaussian filter (s.d. $=50 \mathrm{~ms}$ ). (b) The same neuron in the impatient trials. Trials are sorted in ascending order of the waiting time. PETHs (bottom) of trials grouped by waiting time, as indicated by a color scale. (c) Mean waiting time is plotted against mean firing rate at $0-0.4 \mathrm{~s}$ from poke in. Trials are grouped according to firing rates (4-spikes per s bin) for the visualization purpose only. Error bars represent \pm s.e.m. Circles without error bars represent groups with 1 or 2 trials. Dashed line: regression line. (d) Significance ( $P$ value) of the Pearson's correlation coefficient was calculated at each 0.4-s non-overlapping bin and plotted as a function of time (corrected for the multiple comparisons). Significance level $(P=0.05)$ is indicated by the dashed line. The significance of each time bin is also indicated by the color bar on top. N.S., not significant; N.D., no data.

we estimated the probability of obtaining this result by chance at $P<0.002$ (permutation test, Online Methods).

After selecting a population using relatively strict criteria, we considered in detail further properties of this population that were not included in the selection criteria. Briefly, this population of neurons showed a strong positive or negative correlation between ramp rate and waiting time (Fig. 3d,g and Supplementary Fig. 4), but a small or zero correlation between firing rate at movement time (poke out) and waiting time (Fig. 3e,h). Finally, individual neurons' threshold crossing times predicted future waiting times substantially in the future, up to around $1 \mathrm{~s}$ in advance (Fig. $\mathbf{3 f}$,i).

\section{Transient waiting-time predictive activity}

We next looked for neurons that also showed waiting time predictive activity that did not take the form of ramp-to-threshold. Specifically, we searched for neurons exhibiting different firing rates for different waiting time trials, which could serve as input to an integrator and therefore contribute to the different rates of ramping activity.

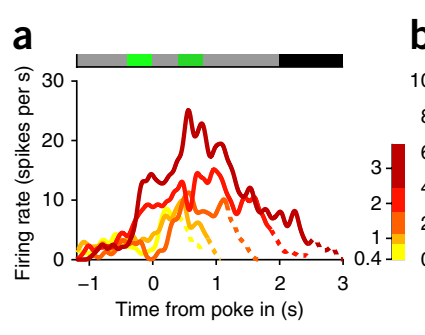

C

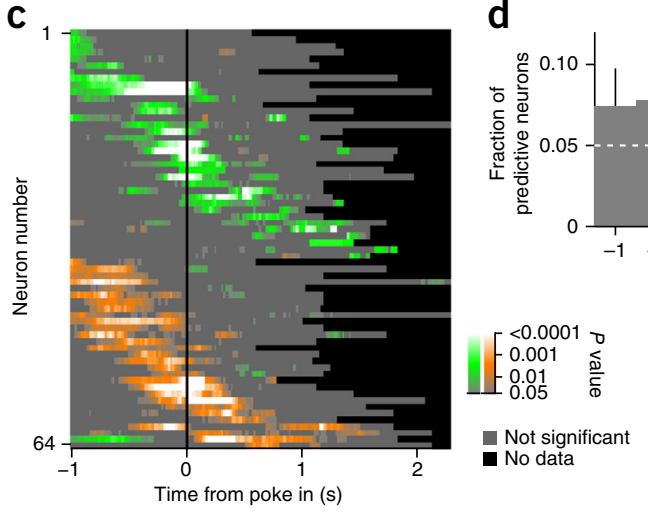

a
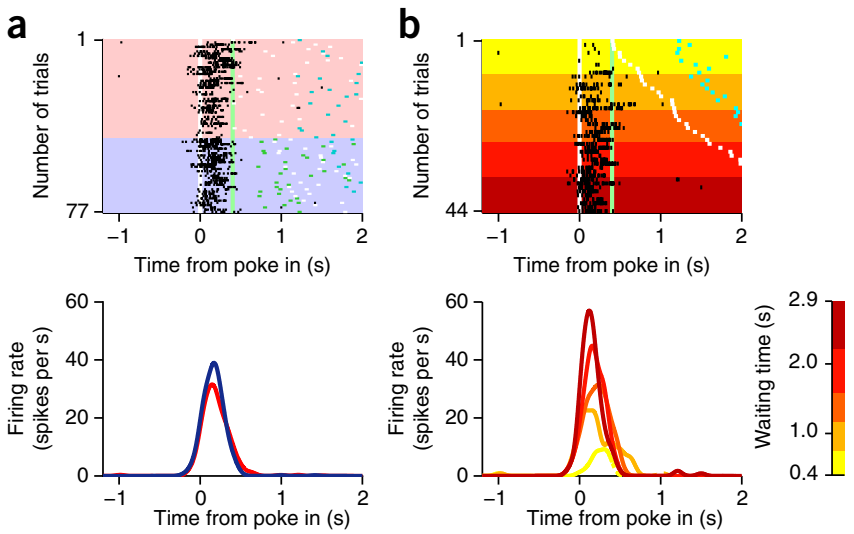

C
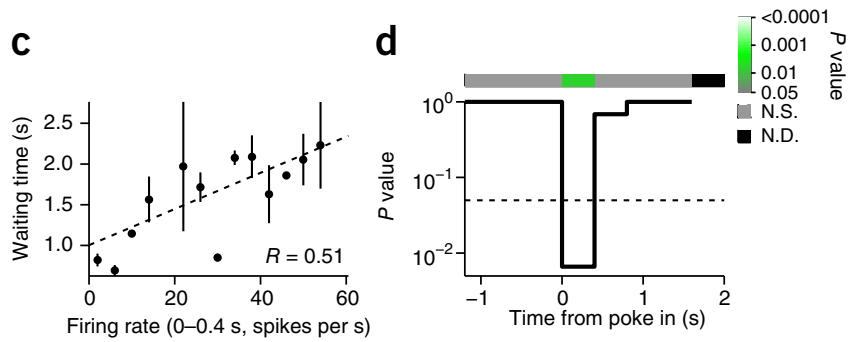

This analysis revealed a larger second population of M2 neurons, an example of which is shown in Figure 4. This neuron showed transient activation when the rat poked into the waiting port (Fig. 4a). The activation was stronger in impatient trials with longer waiting times (Fig. 4b), and the firing rate of this neuron was significantly correlated with waiting time $(R=0.51, P<0.001, n=38$ trials; Fig. 4c). The correlation was significant only in the time window between poke in and tone 1 presentation (Fig. 4d). The activity of this neuron after the poke in was able to predict the waiting time in each trial to the extent of explaining $26 \%$ of the variance.

M2 neurons with transient waiting time correlations showed a variety of different dynamic profiles, including different times and durations of firing. Figure 5a shows an example of a neuron showing a more prolonged activation during the delay period. Figure $\mathbf{5 b}$ shows an example of a neuron with the opposite correlation: waiting time decreased as firing rate increased. Overall, of 356 M2 neurons

Figure 5 Population data of predictive activity. (a,b) Other examples of M2 neurons with predictive activity. (a) Shown is an M2 neuron with sustained activation during waiting and whose firing rate was positively correlated with the waiting time. Data are presented as in Figure $4 \mathbf{b}, \mathbf{d}$. (b) Shown is an M2 neuron with negative correlation between the firing rate and the waiting time. Data are presented as in Figure $\mathbf{4 b , d}$. The color scale in green indicates a positive correlation and orange a negative correlation. (c) Time course and the sign of the correlation for all the predictive neurons ( $n=64$ neurons). The significance of the correlation is calculated for 0.4-s overlapping time window in every 0.02-s time step for each neuron and indicated in each row. Neurons are sorted according to a center of mass of $\log (P$ value $)$. Only the time bins with significant $P$ value were used to calculate the center of mass. The color code is the same as in $\mathbf{b}$. Bonferroni correction for multiple comparisons was used to select neurons with transient correlation, but the $P$ value here is not corrected for the multiple comparisons, as the main points are the time course and the sign of the predictive activity, not its absolute value. (d) Time course of fraction of predictive neurons. For each neuron, waiting time correlation with firing rate was tested on subsamples of 30 impatient trials, allowing comparisons across time bins. Subsampling was performed 1,000 times and error bars represent 95th percentile ranges. The white dashed line indicates chance level of 0.05 . 
a

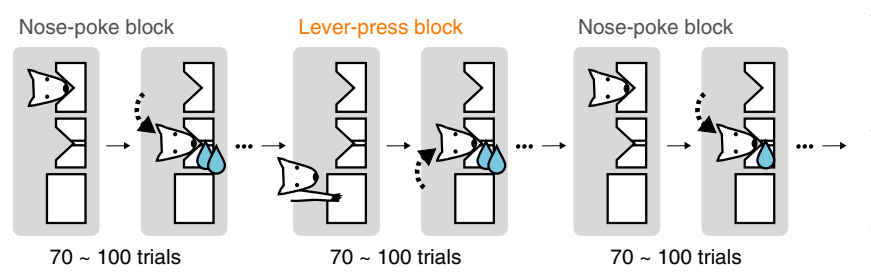

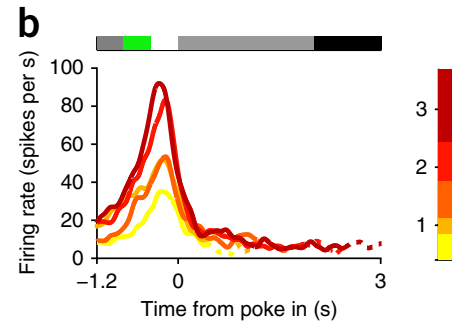

Figure 6 Action specificity of predictive activity. (a) A schematic diagram of a waiting task with interleaved blocks of the nose-poke waiting trials and the lever-press waiting trials (Online Methods). (b) An example of nose poke-specific predictive neurons. PETHs indicate activities of the neuron in the nose-poke waiting trials (left) and in the lever-press waiting trials (right). The color of the bar on top indicates the significance of the time bins. Data are presented as in Figure 4 b,d. (c) The predictive activities of the two types of waiting trials are represented independently in M2. Each circle represents one neuron, indicating the correlation coefficient between the firing rate and the waiting time in the nose-poke trials on the $x$ axis and the correlation coefficient between the firing rate and the waiting time in the lever-press trials on the $y$ axis. The correlation coefficient was calculated at the most significant time bin for each type of trials. examined in a population analysis, $64(18 \%, P<0.002$ with permutation test with trial shuffling) showed transient waiting-time predictive activity (Supplementary Fig. 3 and Online Methods). Across the entire population of waiting time predictive neurons, two features are critical (Fig. 5c). First, different neurons showed predictive activity, spanning the waiting period (Fig. 5c,d). Second, approximately equal numbers of neurons were positively and negatively correlated with waiting time (Fig. 5c). This is consistent with the idea that individual neurons contribute to push waiting time longer or shorter, through either positive or negative coupling to the putative integrator circuit.

\section{Action specificity of waiting-time predictive activity}

If waiting-time predictive activity represents a signal related to preparing a particular action, it should be action specific. Alternatively, predictive activity might represent an abstract signal, such as level of patience or the value of the anticipated outcome of the trial, which would not be expected to be action specific. To differentiate these possibilities, we trained three rats to perform, in addition to the nose-poke
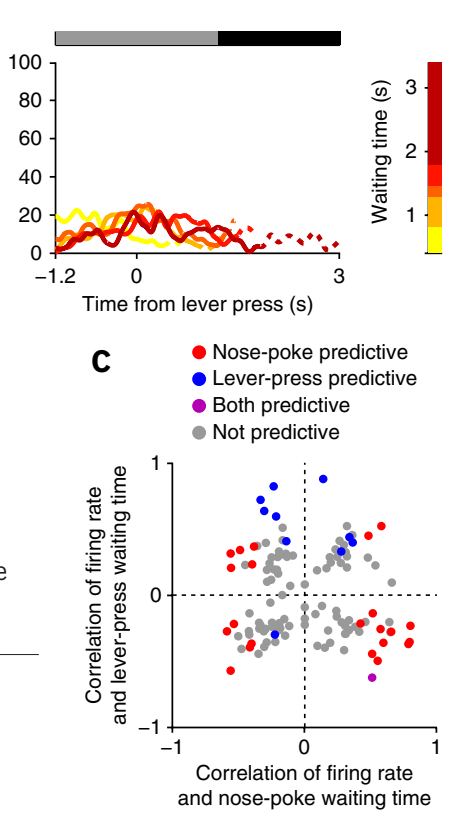

waiting task, a lever-press waiting task (Fig. 6a, Supplementary Fig. 5 and Online Methods) and recorded 175 units in M2 during performance of the dual nose-poke/lever-press waiting task.

An example M2 neuron that showed transient activation in nosepoke trials correlated with waiting time is shown in Figure $\mathbf{6 b}$. The activity of this same neuron was weak during the lever-press trials and was not correlated with lever-press waiting time. To quantify the action specificity of predictive activity in the population of M2 neurons, we compared the strength of correlation between firing rate and waiting time in nose-poke trials with that in lever-press trials (Fig. 6c). The correlation coefficients for those two trial types appeared independent $(R=-0.07, P=0.43$ for all 119 neurons; $R=-0.26, P=0.22$, for all 23 nose-poke predictive neurons; $R=-0.4$, $P=0.18$ for all 11 lever-press predictive neurons). The percentage of lever-press predictive neurons among all the nose-poke predictive
Figure 7 Action specificity of ramp-to-threshold activity. (a) An example of nose poke-specific predictive neurons. PETHs indicate activities of the neuron in the nose-poke waiting trials (left) and in the lever-press waiting trials (right). Data are presented as in Figure 3. (b) Poke-out/delay selectivity index and lever-release/delay selectivity index for all of the nose-poke predictive neurons (ramp-tothreshold type). Of six nose-poke predictive neurons, two neurons did not show significant difference between activity at the lever-release period and activity at the delay period (open circle), and were therefore not tested with threshold-type predictive activity. The other four neurons showed significant difference in activity at the lever-release period and delay period (black and red filled circles), and were therefore tested with the threshold-type predictive activity for the lever-release time. One of them showed significant predictive activity in the lever-press trials, but the direction of ramping activity was the opposite (red filled circle). (c) An example of the lever press-specific predictive neurons. Data are presented as in Figure 3. (d) Lever-release/delay selectivity index and poke-out/delay selectivity index for all the lever-press predictive neurons (ramp-to-threshold type). Of 15 lever-press predictive neurons, nine neurons did not show significant difference between activity at the poke-out period and activity at the delay period (open circle). Six neurons showed significant difference in activity at the poke-out period and delay period (black and red filled circle), and were therefore tested with the threshold-type predictive activity for the poke-out time. One of them (the same as the red neuron in b) showed significant, but opposite, predictive activity in the nose-poke trials.
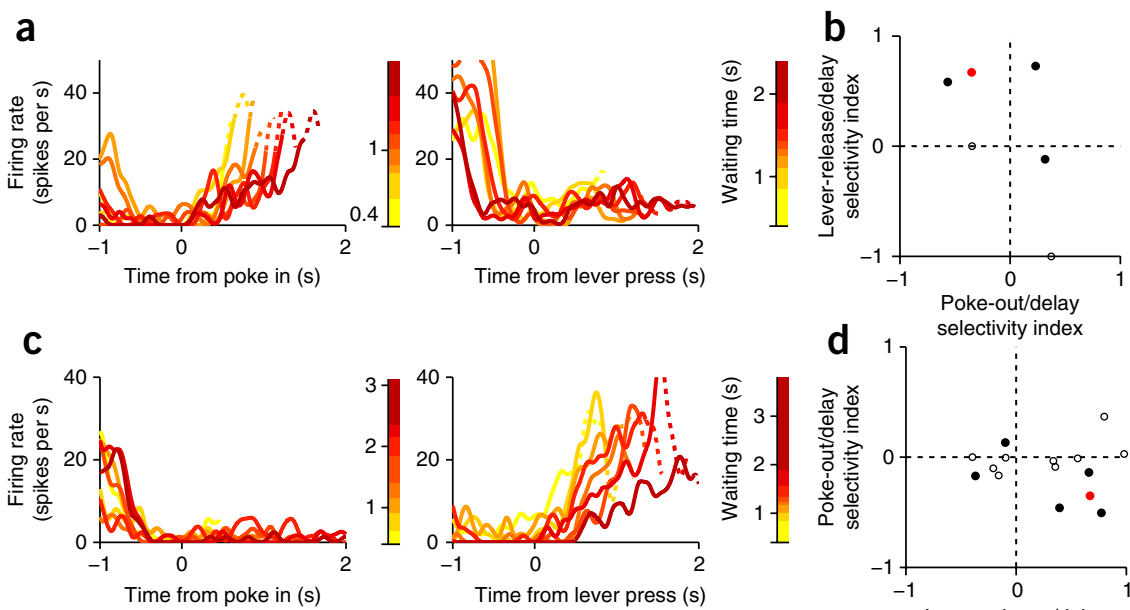

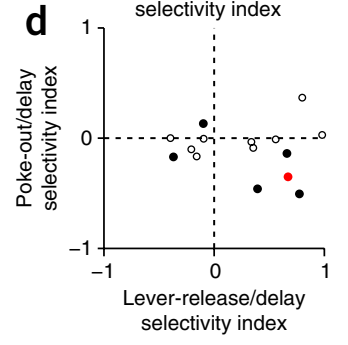


a
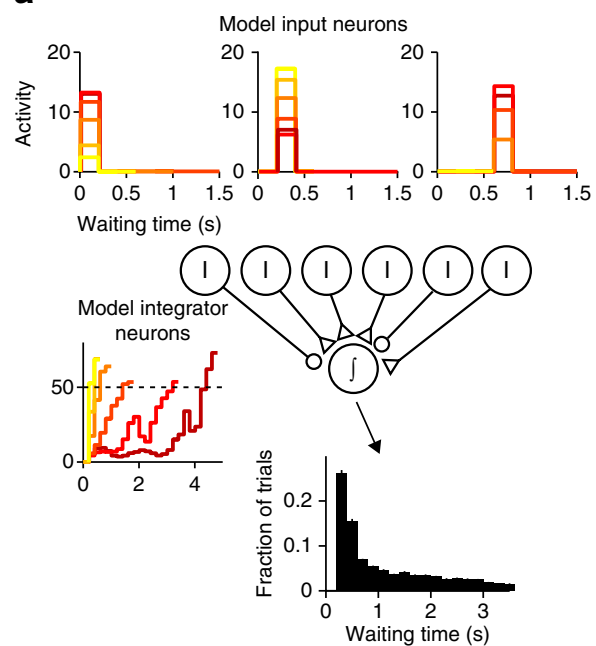

b
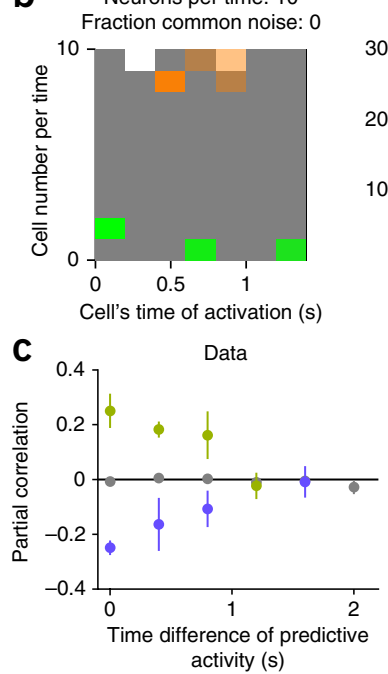

Neurons per time: 300 Fraction common noise: 0

300

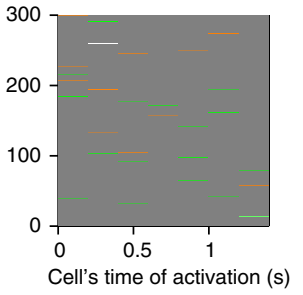

Neurons per time: 300 Fraction common noise: 0.4

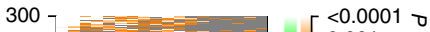

$300]=[0.0001$
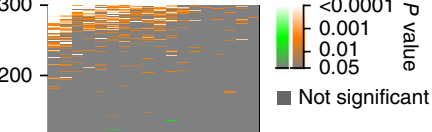

100

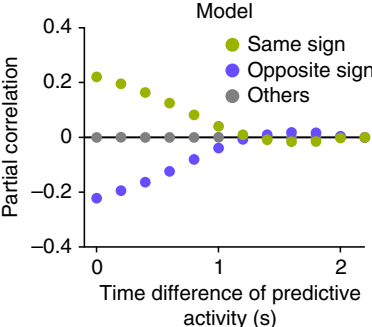

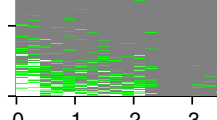

Cell's time of activation (s)

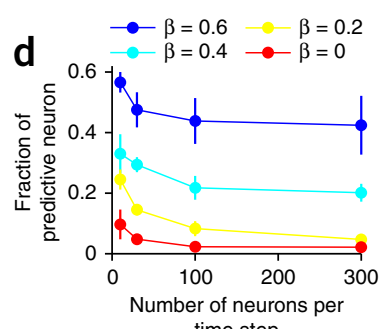

Figure 8 Integrator model. (a) A schematic diagram of an integrator model. Circles with ' $\mathrm{I}$ ' indicate input neurons. A circle with ' $\mathrm{f}$ ' indicates an integrator neuron. A small triangle indicates an excitatory synapse and a small circle indicates an inhibitory synapse. Inset panels show PETHs of example model neurons (top three panels are example input neurons and the bottom left panel is an integrator neuron; data are presented as in Fig. 3a). The bottom right inset panel shows a waiting time histogram of the model (mean \pm s.e.m. of 1,000 model sessions of 100 trials). (b) Waiting time correlation for all the input neurons from three example models with different parameters (number of neurons per time: 10 (left), 300 (middle and right); fraction of common noise ( $\beta$ ): 0 (left and middle), 0.4 (right)). Color indicates $P$ value of waiting time correlation. Neurons are arranged according to its activation time ( $x$ axis) and synaptic weight ( $y$ axis, positive weight at the top and negative weight at the bottom). (c) Left, pairwise partial correlation between simultaneously recorded neurons as a function of time difference of the most predictive time bins of each neuron. Pairs are categorized as the same sign (green), opposite sign (blue) or other (gray) according to the sign of waiting time correlation of each neuron. Error bars indicate s.e.m. $N=1,836$ pairs. Right, pairwise partial correlation between input neurons in the model as a function of time difference of their activities (number of neurons per time, 300; fraction of common noise ( $\beta$ ), 0.4). Mean \pm s.e.m. of 100 model sessions. Error bars are too small to be visible. (d) Fraction of predictive neurons as a function of the number of neurons per time step and fraction of common noise in the input neuron activity $(\beta)$. Mean \pm s.e.m. of 1,000 model sessions.

neurons (4.4\%, 1 of 23 neurons) was not more than would be expected from the percentage of lever-press predictive neurons among all the neurons (11 of 119 neurons, $9.2 \%$; $\chi^{2}$ test, $\chi^{2}(1)=0.81, P=0.37$ ).

We also examined action specificity in neurons with the ramp-tothreshold type predictive activity (Fig. 7). We only found one neuron that showed ramping type predictive activity in both types of waiting trials, but the sign of ramping activity in this neuron was opposite for the two types of trials. These results suggest that the predictive activity in M2 is action specific rather than tied to general states or outcome predictions, although we could not specify whether observed action specificity was a result of effector specificity (nose-poking or leverpressing), action-direction specificity (plan to move left or right) or other forms of specificity. Further characterization of activity of waiting time predictive neurons revealed that weaker predictive activity was already present during the intertrial interval (Supplementary Fig. 6), that activity in patient trials was consistent with activity in impatient trials (Supplementary Fig. 7), and that transient neurons' waitingtime predictive activity can be partly explained by somatic motor input (Supplementary Fig. 8) and trial histories (Supplementary Fig. 9).

\section{Integrator model}

To test whether a neural integration process could explain the relationship between the observed electrophysiology and behavior, we instantiated this theory in a model based on our observations. The input to the integrator consisted of a population of transiently active units connected to an ideal temporal integrator by synaptic weights randomly distributed around a near-zero mean (Fig. 8a). When the integrator reached a given threshold, an action was elicited. Each transient neuron was assigned a specific delay relative to waiting onset, and the amplitude of firing was scaled by a random variable on each trial.

The output of the integration circuit was indeed similar to the activity of the observed ramp-to-threshold neurons (Fig. 8a). The magnitude of activation of transient neurons gained trial-by-trial correlation with waiting times by affecting the slope of ramping activity of the integrator neuron. However, if we assumed that firing of transient neurons were independent of each other, the contribution of each neuron to waiting time decreased markedly as the number of transient neurons increased (Fig. 8b). As it is known that even small inter-neuronal correlations make the responses of individual neurons correlated with behavior even in case of a large population size ${ }^{26}$, we hypothesized that correlations induced by shared input might prevent neural-behavioral correlations from being diluted by the law of large numbers. We first analyzed the actual trial-to-trial firing rate correlations from subsets of simultaneously recorded M2 neurons. To factor out the contribution of waiting time itself to inter-neuronal correlations, we used a partial correlation technique (Online Methods; Fig. 8c). Despite having factored out correlation with waiting time, we observed large inter-neuronal correlations, the pattern of which was such that the relative sign of correlation between neurons and behavioral output determined whether an inter-neuronal correlation will be positive or negative (that is, two neurons that were both positively or negatively correlated with waiting time were on average positively correlated to one another, whereas positive-negative pairs were negatively correlated with one another). Furthermore, the strength of correlation decayed as a function of time difference of the activity of each neuron. To test whether the integration-to-bound model was consistent with these observations, we introduced a common noise source that was injected into all transient units with a sign and strength 
proportional to its synaptic weight (Fig. 8c). By varying the number of neurons and the relative magnitude of shared versus private noise, we characterized the relationship between neural-behavioral and interneuronal correlations. We found that, as the fraction of shared noise was increased, the fraction of transient neurons significantly correlated with waiting time increased, even for large population sizes (Fig. 8d). At a level of common noise sufficient to reproduce the observed neuronal-behavioral correlations, we obtained a prediction for both the magnitude and signs of trial-by-trial correlations between neurons. This prediction was quantitatively consistent with those actually recorded (Fig. 8c). Thus, the integration-to-bound model provided a simple and self-consistent account of the neural-behavioral correlations and inter-neuronal correlations observed in the data.

\section{DISCUSSION \\ Waiting task}

We developed a task in which subjects had to wait for two tones to obtain a large reward, but could respond after the first tone to obtain a small reward. Studies on standard inter-temporal choice tasks ${ }^{11,12}$ have focused on a single choice point at which subjects decide between an immediate and a delayed reward. In contrast, subjects in our task were able to respond for the small reward while waiting, similar to previous human studies 9,10 . As far as we are aware, there are no studies investigating a neural mechanism of spontaneous 'giving up' in such inter-temporal choice procedures. We provide evidence for the involvement of the cortical motor system in such behaviors.

\section{Neural activity and classes}

We recorded two types of neurons whose activity correlated with waiting time, even hundreds of milliseconds to seconds before movement onset. One class of neurons showed ramping activity that reached a threshold just before movement initiation and a ramping rate that was inversely correlated with waiting time. This pattern of activity is reminiscent of neurons in the frontal eye field, premotor and primary motor cortex, lateral intraparietal area, and superior colliculus recorded from monkeys performing sensorimotor tasks $s^{5-7,18,27}$.

The second class of predictive activity occurred transiently and tiled all periods of waiting period and at least $1 \mathrm{~s}$ beforehand. To some degree, they resemble neurons reported in posterior parietal $\operatorname{cortex}^{28}$, medial prefrontal cortex ${ }^{29}$ and striatum ${ }^{30}$. However, unlike those previously described transients carrying signals related to movement location, here the transient neurons carried information about movement timing. Neurons in primate supplementary eye field show a similar form of transient signal predicting reaction time in a stop signal task, but with only about $10 \%$ of the delay and variance that we observed $^{31}$. Although the analyses that we performed identified two classes, we cannot ascertain whether these classes are truly distinct or reflect a spectrum. There was no evidence for segregation of these populations across the surface of M2 (Supplementary Fig. 10) and we were not able to determine whether the profiles segregated by layer. An intriguing possibility is that the transient activity reflects neurons in layer $2 / 3$, as sequences imaged in posterior parietal cortex ${ }^{28}$.

\section{Integration model}

The data supported our hypothesis that action timing could be explained by an integration-to-bound process, a computation hypothesized to underlie choices based on perceptual, mnemonic, value and other forms of decision variables ${ }^{2-4,32,33}$. Our data strengthen the evidence for the applicability of this class of models to cases where there is no evidence per se. In the model, transient waiting-time predictive neurons constitute the input, 'voting', for short or long waiting times.
They fire with different rates from trial-to-trial and are connected to the integrator with positive or negative weights. Ramping neurons, on the other hand, are represented by the output of the integrator. By implementing a model based on this integration-to-bound theory, we were able to compare predictions of the theory to our data. The integration-to-bound model explains a number of salient features of the data parsimoniously. First, it explains the properties of ramping neurons, both the correlation between ramp rate and waiting time and the existence of a uniform threshold at action initiation. It also explains the observation that ramping neurons reach a lower threshold when the rat responds to a tone that arrives while waiting. Second, by using inputs modeled on the observed transient responses, the model explains the neuronal-behavioral correlations between transient neuron activity and waiting time. Notably, despite the existence of correlations, in this model, the decision to act is only made just before movement is observed. The observation of correlations of antecedent neural activity with action timing is a consequence of their causal connection through the integrator and do not imply a decision.

In addition to explaining these observations, the model also led to predictions concerning inter-neuronal correlations that we did not anticipate. We chose to introduce correlations in the model using a single shared noise source. The contribution of the shared noise to each input neuron's firing rate was proportional to the weight of its connection to the integrator. The model predicted that transient neurons must have specific inter-neuronal correlations if individual neurons' correlations with behavior are to be maintained in large pools of neurons.

It is notable that two aspects of this prediction were met. First, the magnitude of shared noise required to achieve the observed fraction of neurons with significant behavioral correlations at population sizes on the order of $10^{3}$ to $10^{4}$ imply a magnitude of inter-neuronal correlations of around 0.2 , as seen in the data. Second, in this model, pairs of neurons with the same sign of behavioral correlations will themselves be positively correlated and pairs of neurons with the opposite sign will be negatively correlated ${ }^{34}$. The model and data also suggest an overall mean correlation level near zero, consistent with balanced network theory ${ }^{35}$. We believe these predictions to be general features of the proposed theory. The biophysical mechanisms supporting integration are an important and unresolved issue. One class of biophysical models suggests that ramping arises from attractor dynamics depending on recurrent excitatory connections ${ }^{2}$. However, we have not tested the predictions of these models. Further elements introduced into the present model, such as variable weighting of inputs have also been considered biophysically ${ }^{36}$. The integration-to-bound mechanism represents a cognitive primitive that may be assembled into diverse computations together with other motifs, such as divisive normalization, spike timing-dependent plasticity or reinforcement learning.

\section{Anatomical context}

As to the neural substrates of the proposed integrator, two general hypotheses could be considered. Because we observed all the constituents of the model in M2, it could be that M2 is capable of supporting integration in local circuitry. Alternatively, integration could occur with a larger circuit comprising a larger network of cortical and subcortical regions to which $\mathrm{M} 2$ is connected. Although the data do not present much evidence bearing on this question, our default is to consider the latter hypothesis to be more likely. One observation favoring this alternative is that ramp-to-threshold activity has already been observed in many other areas $5-7,18,27$. A second argument favoring a widely distributed network is that this would allow more kinds of evidence to be integrated into the same process. Finally, we also conjecture that the slow dynamics that we observed, compared with the much more rapid 
dynamics in stimulus-driven response, would be produced more readily in larger size networks (R. Chaudhuri et al., Soc. Neurosci. Abstr. 597.10, 2012). A specific possibility worth investigating is that integration takes place as a 'reverberation' in the goal-directed circuit including not only M2 (ref. 37), but also ventrolateral orbitofrontal cortex (VLO) 12,23,38, prelimbic cortex ${ }^{39,40}$ and dorsomedial striatum ${ }^{22,38}$.

Rat M2, also known as medial agranular cortex (AGm), and partly overlapping with lateral agranular cortex $\left(\mathrm{AGl}^{22,23}\right)$, is considered on anatomical and physiological grounds to be homologous to the primate higher motor cortices, including premotor cortex, supplementary motor complex and frontal eye field ${ }^{19,22,23}$. At a single-neuron level, M2 (including areas at around anterior-posterior axis (AP) 2-3 mm from Bregma, somewhat more caudal than our center of recordings) contains action predictive choice signals ${ }^{19-21}$. Our recordings spanned a large area of rostral M2 (AP 1.8-5.6, medial-lateral axis (ML) 0.8-2.6 mm from Bregma) and waiting time predictive neurons were present uniformly throughout this extent. The caudal part of M2 partly overlaps with a whisker-related area of medial agranular cortex (AP 0.5-4.5 mm $)^{41,42}$, but the majority of our recordings (70\%) were from a more rostral subregion of M2 (>AP +4.6 mm' Supplementary Fig. 10). Important M2 outputs include primary motor cortex, the dorsocentral region of the striatum and the subthalamic nucleus ${ }^{22}$, as well as brain stem motor nuclei ${ }^{43}$, the superior colliculus and other midbrain motor regions ${ }^{22,43}$. In particular, the superior colliculus, which is involved in orienting decisions in rats ${ }^{44}$, has been proposed to be a key element supporting the threshold nonlinearity in integration ${ }^{45}$.

\section{Origin of trial-by-trial fluctuations}

In our task, waiting times exhibited near Poisson variability. To account for this variability with the integration-to-bound model, it was necessary to introduce large amounts of noise in model units. We do not take this as evidence for noise introduced by the brain, but rather as a call for further scrutiny of the contributors to this variability. One source of variability in trial-by-trial neural activity might be variability in movements or posture during waiting. To test this, we performed video tracking and extracted time series of position and orientation of the rat's body. Although the rats' behavior during waiting was relatively constrained by the requirement of keeping the snout inside the waiting port (or paw on the lever), multilinear regression analysis showed that around 20\% of waiting time predictive neurons activity could be explained by these features, whereas 80\% remained significant (Supplementary Fig. 8). We consider it plausible that transients reflect input from sensory areas, especially somatosensory and proprioceptive input, given the strong connections between motor cortices and somatosensory cortices.

A recent anatomical study suggested the existence of an entire body map in the rodent M2 (ref. 46). We did not determine precisely the somatic locations linked to the recording locations, but the wide distribution of waiting time correlated activity suggests the recruitment of multiple body areas in this task. Specific micro-movements may have been incidentally reinforced, leading to 'superstitious' behavior (R. Kawai et al., Soc. Neurosci. Abstr. 790.1, 2010) and amplifying the trial-to-trial variability. In addition to these somatic-motor loops, we could expect additional contributions to transient fluctuations from interoceptive systems and other sensory systems. Under the hypothesis that M2 is a general locus of integrated signals, in tasks in which reward is contingent on specifically controlled sensory input, we would expect to observe similar ramping activity, but with inputs dominated by the appropriate sensory modalities.

\section{Value and intertemporal choice}

Another likely source of variability in waiting time predictive neurons is variability in decision values originated from the past trial history of waiting decisions and reward outcomes. Waiting and responding were associated with different decision outcomes (small versus large water amount) and different costs (waiting time), which might be updated through experience. Waiting-time predictive neurons might therefore reflect, in part, decision values as well as action plans ${ }^{21}$. Although substantial evidence against abstract value coding is provided by our experiment showing that M2 neurons are strongly selective for specific action sequences, even when they were associated with similar reward sizes and waiting times (Fig. 6), it is possible that the decision value associated with specific actions is multiplexed with waiting time predictive signals. A multiple regression analysis did reveal a contribution of past trial decisions and outcomes to activity in subsequent trials, but this reflected only $9 \%$ of the trial-by-trial variance. Small increments and decrements in activity according to the trial history would allow subjects to adjust their mean waiting time in the face of changing motivational conditions. Finally, from these considerations, we infer that other functionally relevant variables may gain access to influencing M2 input, thereby suggesting a hypothesis for how confidence signals ${ }^{47}$ may be read out by waiting time $e^{48}$.

\section{Implications for self-initiated actions}

Slow building activity has been theorized for nearly 50 years as a neural mechanism for generation of self-initiated actions ${ }^{13-17}$. Here, we have strengthened these concepts in four dimensions. First, our data strengthen the evidence that an observer can 'forecast' a simple decision by observing the state of the brain before a decision on a single trial basis ${ }^{17,18}$. Second, our results provide the strongest level of quantitative evidence supporting the involvement of an integration-to-bound mechanism. Notably, our model predicted previously unobserved patterns of inter-neuronal correlation. Third, these recordings provide compelling evidence linking neurons in the rat premotor cortex to neural activity observed both in human electroencephalogram (readiness potential ${ }^{13,14}$ ) and primate frontal cortices ${ }^{15-18}$. These cortices are considered functionally homologous to rodent M2 (refs. 22,23). Our experiments provide a rodent model of an important cognitive phenomenon with physiological and anatomical justification and extend the applicability of this class of experiments across species. Fourth, these results identify, to the best of our knowledge for the first time, a possible driving force or causal antecedent for ramping activity (that is, transient input neurons; Figs. 4 and 5). This finding strengthens the plausibility of the integration-to-bound model to the case of voluntary actions. It also opens doors to further studies to elucidate the properties of these antecedent signals and dissect neural mechanisms underlying concepts such as 'will' and 'self' that are used to describe this class of actions.

Supported by these considerations, an integration-to-bound theory of voluntary action provides a potential resolution to the long-standing controversy over the interpretation of previous experiments showing that readiness potentials precede the subjective conscious intention to move $\mathrm{e}^{14}$. The theory explains how activity preceding bound crossing, either input or accumulated activity, can be said to participate causally in the timing of an action, but does not uniquely specify it. The integration-to-bound theory implies that no decision has been made until the bound has been reached. Thus, assuming a threshold above the size of an individual input, more than one individual input must occur to reach a decision; no individual neuron contributing to the integrator is a unique cause.

The crossing of an arbitrary lower threshold by ramping activity ${ }^{13,14}$ may partially forecast action, but logically it cannot fully predict action, as at any moment up to bound crossing the arrival of opposing inputs may avert an action no matter how strongly it is forecasted ${ }^{49}$. In contrast, after reaching the action bound, an action is inevitable. Thus, sub-bound activity may be associated with predictive, causal activity of a qualitatively 
different nature than super-bound activity, similar to a proposed distinction between subconscious and conscious neural processes ${ }^{50}$. The relative delay between threshold crossing and movement initiation observed in rat M2 (around $150 \mathrm{~ms}$ ) were consistent with the delay between human subjects' awareness of their intention to act and movement (around $200 \mathrm{~ms})^{14}$. It could therefore be inferred that crossing the threshold from unawareness to awareness is a reflection of bound crossing. In this way, the integration-to-bound theory may help to resolve the contradiction between the subjective report of free will and the requirement for causal antecedents to non-capricious, willed actions. Finally, insofar as simple voluntary actions constitute an appropriate experimental context, our results provide a starting point for investigating mechanisms underlying concepts such as self, will and intention to act, which might be conserved among mammalian species.

\section{METHODS}

Methods and any associated references are available in the online version of the paper.

\section{Note: Any Supplementary Information and Source Data files are available in the online version of the paper.}

\section{ACKNOWLEDGMENTS}

We thank the members of the Mainen laboratory for discussion, M. Terrelonge for assistance with recording experiments, B. Burbach and M. Vinhas for technical assistance, E. Lottem for daily discussions, and C. Feierstein, H. Shteingart, Y. Loewenstein, J. Erlich, J. Paton and B. Atallah for helpful comments on the manuscript. This work was supported by the Uehara Memorial Foundation (M.M.), Fundação Bial (127/08, M.M.), Fundação para a Ciência e a Tecnologia SFRH/ BPD/46314/2008, M.M.; SFRH/BD/33274/2007, M.I.V.; SFRH/BD/32947/2006, G.M.C.), European Research Council Advanced Investigator Grant (250334, Z.F.M.) and Champalimaud Foundation (Z.F.M.). G.M.C. was supported by Fundação para a Ciência e a Tecnologia, as part of the $\mathrm{BEB} / \mathrm{CNC} \mathrm{PhD}$ programme.

\section{AUTHOR CONTRIBUTIONS}

M.M. and Z.F.M. designed the experiments, analyses and models and wrote the manuscript. M.M. conducted the experiments with assistance from M.I.V. and G.M.C. M.M. analyzed the data and implemented the model.

\section{COMPETING FINANCIAL INTERESTS}

The authors declare no competing financial interests.

Reprints and permissions information is available online at http://www.nature.com/ reprints/index.html.

1. Uchida, N., Kepecs, A. \& Mainen, Z.F. Seeing at a glance, smelling in a whiff: rapid forms of perceptual decision making. Nat. Rev. Neurosci. 7, 485-491 (2006).

2. Wang, X.J. Probabilistic decision making by slow reverberation in cortical circuits. Neuron 36, 955-968 (2002).

3. Mazurek, M.E., Roitman, J.D., Jochen, D. \& Shadlen, M.N. A role for neural integrators in perceptual decision making. Cereb. Cortex 13, 1257-1269 (2003).

4. Gold, J.I. \& Shadlen, M.N. The neural basis of decision making. Annu. Rev. Neurosci. 30, 535-574 (2007).

5. Hanes, D.P. \& Schall, J.D. Neural control of voluntary movement initiation. Science 274, 427 (1996).

6. Ratcliff, R., Cherian, A. \& Segraves, M. A comparison of Macaque behavior and superior colliculus neuronal activity to predictions from models of two-choice decisions. J. Neurophysiol. 90, 1392-1407 (2003).

7. Roitman, J.D. \& Shadlen, M.N. Response of neurons in the lateral intraparietal area during a combined visual discrimination reaction time task. J. Neurosci. 22, 9475-9489 (2002).

8. Berns, G.S., Laibson, D. \& Loewenstein, G. Intertemporal choice-toward an integrative framework. Trends Cogn. Sci. 11, 482-488 (2007).

9. McGuire, J.T. \& Kable, J.W. Rational temporal predictions can underlie apparent failures to delay gratification. Psychol. Rev. 120, 395-410 (2013).

10. Mischel, W., Ebbesen, E.B. \& Zeiss, A.R. Cognitive and attentional mechanisms in delay of gratification. J. Pers. Soc. Psychol. 21, 204-218 (1972).

11. Cardinal, R.N., Winstanley, C.A., Robbins, T.W. \& Everitt, B.J. Limbic corticostriatal systems and delayed reinforcement. Ann. NY Acad. Sci. 1021, 33-50 (2004).

12. Roesch, M.R., Calu, D.J., Burke, K.A. \& Schoenbaum, G. Should I stay or should I go? Transformation of time-discounted rewards in orbitofrontal cortex and associated brain circuits. Ann. NY Acad. Sci. 1104, 21-34 (2007).
13. Deecke, L., Scheid, P. \& Kornhuber, H.H. Distribution of readiness potential, premotion positivity and motor potential of the human cerebral cortex preceding voluntary finger movements. Exp. Brain Res. 7, 158-168 (1969).

14. Libet, B., Gleason, C.A., Wright, E.W. \& Pearl, D.K. Time of conscious intention to act in relation to onset of cerebral activity (readiness-potential). Brain 106, 623-642 (1983).

15. Okano, K. \& Tanji, J. Neuronal activities in the primate motor fields of the agranular frontal cortex preceding visually triggered and self-paced movement. Exp. Brain Res. 66, 155-166 (1987).

16. Romo, R. \& Schultz, W. Role of primate basal ganglia and frontal cortex in the internal generation of movements. III. Neuronal activity in the supplementary motor area. Exp. Brain Res. 91, 396-407 (1992).

17. Fried, I., Mukamel, R. \& Kreiman, G. Internally generated preactivation of single neurons in human medial frontal cortex predicts volition. Neuron 69, 548-562 (2011).

18. Lebedev, M.A., O'Doherty, J.E. \& Nicolelis, M.A.L. Decoding of temporal intervals from cortical ensemble activity. J. Neurophysiol. 99, 166-186 (2008).

19. Erlich, J.C., Bialek, M. \& Brody, C.D. A cortical substrate for memory-guided orienting in the rat. Neuron 72, 330-343 (2011).

20. Guo, Z.V. et al. Flow of cortical activity underlying a tactile decision in mice. Neuron 81, 179-194 (2014).

21. Sul, J.H., Jo, S., Lee, D. \& Jung, M.W. Role of rodent secondary motor cortex in value-based action selection. Nat. Neurosci. 14, 1202-1208 (2011).

22. Reep, R.L., Corwin, J. \& Hashimoto, A. Efferent connections of the rostral portion of medial agranular cortex in rats. Brain Res. Bull. 19, 203-221 (1987)

23. Reep, R.L. \& Goodwin, G. Topographic organization in the corticocortical connections of medial agranular cortex in rats. J. Comp. Neurol. 294, 262-280 (1990).

24. Jaramillo, S. \& Zador, A.M. The auditory cortex mediates the perceptual effects of acoustic temporal expectation. Nat. Neurosci. 14, 246-251 (2011).

25. Lauwereyns, J., Watanabe, K., Coe, B. \& Hikosaka, O. A neural correlate of response bias in monkey caudate nucleus. Nature 418, 413-417 (2002).

26. Zohary, E., Shadlen, M.N. \& Newsome, W.T. Correlated neuronal discharge rate and its implications for psychophysical performance. Nature 370, 140-143 (1994).

27. Maimon, G. \& Assad, J.A. A cognitive signal for the proactive timing of action in macaque LIP. Nat. Neurosci. 9, 948-955 (2006)

28. Harvey, C.D., Coen, P. \& Tank, D.W. Choice-specific sequences in parietal cortex during a virtual-navigation decision task. Nature 484, 62-68 (2012).

29. Fujisawa, S., Amarasingham, A., Harrison, M.T. \& Buzsáki, G. Behavior-dependent short-term assembly dynamics in the medial prefrontal cortex. Nat. Neurosci. 11, 823-833 (2008).

30. Lau, B. \& Glimcher, P.W. Action and outcome encoding in the primate caudate nucleus. J. Neurosci. 27, 14502-14514 (2007).

31. Stuphorn, V., Brown, J.W. \& Schall, J.D. Role of supplementary eye field in saccade initiation: executive, not direct, control. J. Neurophysiol. 103, 801-816 (2010).

32. Milosavljevic, M., Malmaud, J. \& Huth, A. The drift diffusion model can account for the accuracy and reaction time of value-based choices under high and low time pressure. Judgm. Decis. Mak. 5, 437-449 (2010).

33. Ratcliff, R. A theory of memory retrieval. Psychol. Rev. 85, 59-108 (1978).

34. Machens, C.K., Romo, R. \& Brody, C.D. Flexible control of mutual inhibition: a neural model of two-interval discrimination. Science 307, 1121-1124 (2005).

35. Renart, A. et al. The asynchronous state in cortical circuits. Science 327, 587-590 (2010).

36. Soltani, A. \& Wang, X.-J. Synaptic computation underlying probabilistic inference. Nat. Neurosci. 13, 112-119 (2010).

37. Gremel, C.M. \& Costa, R.M. Premotor cortex is critical for goal-directed actions Front. Comput. Neurosci. 7, 110 (2013).

38. Gremel, C.M. \& Costa, R.M. Orbitofrontal and striatal circuits dynamically encode the shift between goal-directed and habitual actions. Nat. Commun. 4, 2264 (2013).

39. Narayanan, N.S. \& Laubach, M. Top-down control of motor cortex ensembles by dorsomedial prefrontal cortex. Neuron 52, 921-931 (2006).

40. Botvinick, M.M., Cohen, J.D. \& Carter, C.S. Conflict monitoring and anterio cingulate cortex: an update. Trends Cogn. Sci. 8, 539-546 (2004).

41. Brecht, M. et al. Organization of rat vibrissa motor cortex and adjacent areas according to cytoarchitectonics, microstimulation, and intracellular stimulation of identified cells. J. Comp. Neurol. 479, 360-373 (2004).

42. Neafsay, E.J. et al. The organization of the rat motor cortex: a microstimulation mapping study. Brain Res. 396, 77-96 (1986)

43. Stuesse, S.L. \& Newman, D.B. Projections from the medial agranular cortex to brain stem visuomotor centers in rats. Exp. Brain Res. 80, 532-544 (1990).

44. Felsen, G. \& Mainen, Z.F. Neural substrates of sensory-guided locomotor decisions in the rat superior colliculus. Neuron 60, 137-148 (2008).

45. Lo, C.-C. \& Wang, X.-J. Cortico-basal ganglia circuit mechanism for a decision threshold in reaction time tasks. Nat. Neurosci. 9, 956-963 (2006).

46. Zingg, B. et al. Neural networks of the mouse neocortex. Cell 156, 1096-1111 (2014)

47. Kepecs, A., Uchida, N., Zariwala, H.a \& Mainen, Z.F. Neural correlates, computation and behavioural impact of decision confidence. Nature 455, 227-231 (2008).

48. Lak, A. et al. Orbitofrontal cortex is required for optimal waiting based on decision confidence. Neuron (in the press).

49. Schall, J.D. Neural basis of deciding, choosing and acting. Nat. Rev. Neurosci. 2 , 33-42 (2001).

50. Dehaene, S., Changeux, J.-P., Naccache, L., Sackur, J. \& Sergent, C. Conscious, preconscious, and subliminal processing: a testable taxonomy. Trends Cogn. Sci. 10, 204-211 (2006). 


\section{ONLINE METHODS}

Animal subjects. All procedures involving animals were either carried out in accordance with US National Institutes of Health standards and approved by Cold Spring Harbor Laboratory Institutional Animal Care and Use Committee or in accordance with European Union Directive 86/609/EEC and approved by Direcção-Geral de Veterinária. Experiments were performed on 37 male adult Long-Evans hooded rats. Rats had free access to food, but water was restricted to the behavioral session and 20-30 additional min per $\mathrm{d}$.

Behavioral task. Rats were trained and tested on the waiting task (Fig. 1a,b). In the standard waiting task, the behavioral box contained a wall with three ports (Island Motion) $)^{44}$. The waiting port was located at the center and the reward port was located at the side. The side of the reward port was chosen randomly for each rat. The third port was inactive. Entry to and exit from the ports were detected based on an infrared photo-beam located inside each port.

Rats initiated a trial by poking their snout into the waiting port (Supplementary Video 1). The first tone, tone 1 (6- or $14-\mathrm{kHz}$ tone, $80 \mathrm{~ms}$ ) was played, if the rat kept its snout inside the waiting port for T1 delay $(0.4 \mathrm{~s})$. Tone 1 signaled the availability of a small amount of water reward at the reward port. If the rat moved out before tone 1 , no rewards were available in that trial (short poke trial). If the rat moved out of the waiting port after tone 1 and visited the reward port, a small water reward $(10 \mu \mathrm{l})$ was delivered through a tube in that port after a 0.5 -s delay (impatient trial). If the rat did not respond to tone 1 and waited with its snout in the waiting port, a second tone, tone $2(14-$ or $6-\mathrm{kHz}$, differing from tone $1,80 \mathrm{~ms}$ ) was played after a certain delay (T2 delay). If the rat visited the reward port after tone 2 , a large reward $(40 \mu \mathrm{l})$ was delivered after a 0.5 -s delay (patient trial). For three of the rats, the reward sizes were different: $14 \mu \mathrm{l}$ for the small and $30 \mu \mathrm{l}$ for the large rewards was used. There were no signals to the rat that it had exited the waiting port. Re-entrance to the waiting port (multi-poke) was signaled by brief noise burst $(60 \mathrm{~ms})$ to discourage this behavior. Because we did not know whether the rat intended to leave the waiting port or just failed to wait unintentionally in the multi-poke trials, we excluded this type of trials from the neural analysis. The rat also had to visit the reward port in $2 \mathrm{~s}$ after the initial poke out to collect rewards, and trials violating this requirement were also excluded from neural analysis.

Waiting time was defined as a time from the entry into the waiting port to the movement out of the waiting port (Fig. 1a). As a measure for trial-by-trial variability, we calculated average $\mathrm{CV} 2$ of the interval between waiting time and T1 delay for each rat. CV2 was calculated for each 2 consecutive impatient trials as

$$
2\left|I_{n}-I_{n-1}\right| /\left(I_{n}+I_{n-1}\right)
$$

where $I_{n}$ denotes an interval between waiting time and T1 delay in $n$th trial $^{51}$. If leaving the port after the tone 1 is a Poisson process, the expected value is 1 . If waiting time is constant or slowly changing across trials, the value should be close to 0 .

T2 delay was drawn randomly from an exponential distribution, whose minimum value was $0.7 \mathrm{~s}$ and whose mean value was adjusted according to the performance of the animal so that rats succeeded in waiting on about one third of trials. For 33 rats, including 5 rats used in M2 recordings, the mean value of the exponential distribution was adjusted every trial; after each short-poke trial or impatient trial, the mean was decreased by $20 \mathrm{~ms}$. After each patient trial, the mean was increased by $40 \mathrm{~ms}$. For 4 rats, including 3 rats used in M2 recordings, the mean value was set manually for each behavioral session and stayed constant for the entire session. We pooled data from the two conditions, because behavioral and neural recording data showed similar trends in both conditions (data not shown).

Response time to tone 2 was defined as time from the onset of tone 2 to the poke out (Fig. 1a). A histogram of the response time was generated from the data pooled across sessions in each rat. To test whether the rat was responding to tone 2 , we shuffled tone 2 delays from both the impatient and patient trials in each session and made a new response time histogram from the patient trials of shuffled data. We repeated this procedure 1,000 times to estimate the $95 \%$ confidence interval. The peak of the data histogram was defined as significant if the peak bin (20-ms bin size) and one of the adjacent bins were above the $95 \%$ confidence interval of the shuffled histogram. Movement time was defined as the time from leaving the waiting port to entering the reward port (Fig. 1a). To compare movement times from impatient and patient trials without the con- tribution of difference in waiting time distribution (Fig. 1c), we selected subsets of impatient and patient trials to match the waiting time distributions from the two trial types. For this purpose, we first selected the single shortest waiting time trial with waiting time $>700 \mathrm{~ms}$. Then we selected the rest of the trials so that if we sorted all the selected trials according to the waiting time, the order of the trial types would alternate: that is, impatient (Imp), patient (Pat), Pat, Imp, Imp, Pat, and so on, if the first selected trial was an impatient trial (if the first selected trial was a patient trial, then the opposite). With this selection method, $37.7 \pm$ $0.4 \%$ (329 sessions from 37 rats) of trials was selected.

An intertrial interval (ITI) period started after the delivery of the reward. During the ITI period, a white noise was played. The time from the initial poke into the waiting port to the end of the ITI was constant, so that the rat could not profit from leaving the waiting port early to start the next trial early. Thus, the optimal strategy to obtain maximal reward in this task was always to wait for tone 2 .

To test whether neuronal responses were specific for a given action, 3 rats were trained on two variants of the waiting task. For these experiments, the behavioral box contained a wall with a reward port at the center flanked by a nose-poke waiting port on one side and a lever-press waiting port on the other side (Fig. 6a). The rat was required to perform interleaved blocks of nose-poke waiting trials and lever-press waiting trials in a single session. In the nose-poke block, the rat was required to perform the same task as described previously. In a lever-press block, the rule of the task was the same except that the rat had to wait for the tones by keeping the lever pressed. Lever-press waiting in the nose-poke block (or vice versa) was not rewarded. Each block consisted of 70-100 trials. Transitions between the blocks were not signaled explicitly.

Neural recording. For the recording experiments, each rat was implanted with a drive (Island Motion) containing 10-24 movable tetrodes ${ }^{44}$ targeted to the M2 (3.2-4.7 mm anterior to and $1.5-2.0 \mathrm{~mm}$ lateral to Bregma ${ }^{52}$ ) contralateral to the side of the reward port. In case of a dual waiting task in Figure 6, the drive was implanted contralateral to the side of the reward port in a nose-poke waiting task. Rats were allowed to recover for $5 \mathrm{~d}$ before water restriction resumed and the recording sessions began.

Individual tetrodes consisted of four twisted polyimide-coated nichrome wires (H.P. Reid; single-wire diameter $=12.5 \mu \mathrm{m}$ ) gold-plated to $0.2-0.5-\mathrm{M} \Omega$ impedance at $1 \mathrm{kHz}$. The tetrodes were coated with DiI (Molecular Probes) to visualize the tetrode tracks in a histological examination. Electrical signals were amplified and recorded using the NSpike data acquisition system (L.M. Frank, University of California, San Francisco, and J. MacArthur, Harvard University Electronic Instrument Design Lab). Multiple single units were isolated offline by manually clustering spike features derived from the waveforms of recorded putative units using MCLUST software (A.D. Redish, University of Minnesota). Tetrode depths were adjusted before or after each recording session in order to sample an independent population of neurons across sessions. Single-units recorded during more than one session, as judged from the spike waveform and the firing pattern, were excluded from the analysis. The locations of tetrode tips during each recording session were estimated based on their depth and histological examination based on electrolytic lesions and the visible tetrode tracks (Supplementary Fig. 10). Single-units recorded from outside the M2 were excluded from the analysis. Rats performed 1 session per day, and a total of 135 recording sessions were obtained from 8 rats.

Histology. To verify the ultimate location of the tetrodes, electrolytic lesions were produced after the final recording session ( $15 \mu \mathrm{A}$ of cathodal current, $10 \mathrm{~s})$. Rats were then deeply anesthetized with pentobarbital and perfused transcardially with $4 \%$ paraformaldehyde (wt/vol). The brain was sectioned at $50 \mu \mathrm{m}$. Every other slice was stained with Cresyl violet solution to observe sites of electrolytic lesions. Other slices were prepared for fluorescent observation to examine the fluorescent tracks made by DiI-coated tetrodes.

Neural data analysis. All data analysis was performed with custom-written software using MATLAB (Mathworks). No statistical methods were used to pre-determine sample sizes. But our sample sizes were similar to those reported in previous studies ${ }^{19,21}$. Two-sided tests were used for all the statistical tests. All the PETHs were smoothed with a Gaussian filter (s.d. = $50 \mathrm{~ms}$ ) only for visualization purpose. 
For the ramp-to-threshold analysis in Figure 3, only impatient trials were analyzed because in patient trials, the response was triggered by the tone. Only those neurons which showed different firing rates during the delay period (from $0.4 \mathrm{~s}$ after poke in to $0.4 \mathrm{~s}$ before poke out) and the poke-out period (last $0.4 \mathrm{~s}$ before poke out) were analyzed (Wilcoxon signed-rank text, $P<0.01$, ramp-up or ramp-down neurons). Neurons with less than ten long waiting trials (more than $1.2 \mathrm{~s}$ waiting time) were pre-excluded before the selection process because the delay period firing rate could not be estimated reliably. Impatient trials were divided into ten groups based on the waiting time, with equal (or different by 1 ) number of trials per group. Spike trains were smoothed with a causal filter (EPSP-like filter, which is a multiple of two exponentials, one rising exponential with $\tau=1 \mathrm{~ms}$, and the other falling exponential with $\tau=40 \mathrm{~ms}$ ) to generate a PETH for each group. The threshold crossing time was determined from the PETHs. While ramp-up neurons were tested with threshold crossing with positive slope, ramp-down neurons were tested with negative slope. Threshold crossing time was detected when the PETH first crossed the threshold and stayed above (or below for ramp-down neuron) the threshold for more than $20 \mathrm{~ms}$. The threshold crossing time was defined as the end point of this $20 \mathrm{~ms}$. The search for threshold crossing was started at the trough time between 0 and $0.4 \mathrm{~s}$ from the poke in (peak time for the ramp-down neurons) of the average of the ten PETHs and ended at $0.4 \mathrm{~s}$ after the waiting time of the longest waiting time trial. For each neuron, we tested ten different firing rate thresholds, determined as follows: The lowest threshold was set at the lowest firing rate at which threshold crossing occurred in at least 9 out of 10 PETHs. The highest threshold was set at the highest firing rate at which threshold-crossing occurred at least in 9 PETHs. Eight intermediate thresholds were equally-spaced in between the lowest and the highest thresholds. At each threshold level, we calculated correlation between the mean waiting times against the threshold crossing times. A ramp-to-threshold type predictive neurons was defined as a neuron which showed, significant correlation coefficient between the threshold crossing time and the waiting time in at least 2 of the 10 thresholds (corrected for multiple comparisons; two significant thresholds were required for the calculation of rate of ramping explained later), positive prediction time (defined below) in at least 2 significant thresholds, and the regression slope for threshold crossing time against the waiting time close to unity (value between 0.8 and 1.2) in at least 1 significant threshold with positive prediction time. Prediction time was defined for each threshold as an average of waiting time minus time to cross threshold across different waiting time groups. The earliest prediction time was defined for each neuron as a prediction time calculated at the lowest significant threshold for ramp-up neurons (the highest significant threshold for ramp-down neurons). For each PETH, rate of ramping was calculated as a slope of a line connecting 2 points. One was the point of crossing the highest significant threshold with near unity slope and the other was the point of crossing the lowest significant threshold (opposite for ramp-down neurons). For ramp-down neurons log ramp rate is calculated as follows: [log ramp rate] = $-\log (\mid$ ramp rate|). To estimate the significance of fraction of ramp-to-threshold type neurons, we ran a permutation test by randomly permuting the waiting times and neural data across impatient trials. We repeated this procedure 1,000 times to estimate the probability of obtaining the observed fraction of significant neurons by chance. To obtain the correlation coefficient between activity at the poke-out period and the waiting time in Figure $\mathbf{3 e}, \mathbf{h}$, firing rates during the last $0.05 \mathrm{~s}$ before poke out was used.

For the analysis of correlation between the firing rate and the waiting time in Figure 4, we excluded 27 ramp-to-threshold type neurons, because in this analysis, we looked for a different class of waiting-time predictive activity from the ramp-to-threshold type neurons. Only impatient trials were analyzed because in patient trials, the response was triggered by the tone. Two neurons with fewer than 10 impatient trials were excluded from the analysis. The correlation coefficient between the firing rate and the waiting time was examined for nonoverlapping 0.4-s time window, starting from $1.2 \mathrm{~s}$ before the waiting port entry. For each time window, the trials in which the rat had already exited the waiting port by the end of the analysis time window or within $0.2 \mathrm{~s}$ after the end of the time window were excluded from the analysis, in order to exclude potential movement correlates. Time windows with less than 10 trials after the exclusion were not analyzed, because the correlation could not be estimated reliably. Because the significance was tested in multiple time windows, we corrected the significance level using Bonferroni correction. To estimate the significance of fraction of firing rate correlated neurons, we ran a permutation test by shuffling the impatient trials 1,000 times.

For the analysis of the action specificity of the predictive activity in Figure 6, we excluded 35 neurons which was recorded in fewer than 10 impatient trials in either nose-poke or lever-press waiting task. We further excluded 21 neurons which showed ramp-to-threshold type activity in either nose-poke or lever-press waiting tasks to be consistent with the analysis for Figures $\mathbf{4}$ and $\mathbf{5}$. But analysis without exclusion yielded similar results (data not shown).

For the analysis of action specificity of ramp-to-threshold activity in Figure 7, poke-out/delay selectivity index was defined for nose-poke trials as the difference in a mean firing rate during poke-out period (last $400 \mathrm{~ms}$ before poke out) and during the delay period (from $400 \mathrm{~ms}$ after poke in to $400 \mathrm{~ms}$ before poke out) normalized by the sum of the two. The index ranges from -1 to $1 ; 1$ indicates a neuron selective for poke-out period. The lever-release/delay selectivity index was defined as a similar manner for lever-press trials.

To explore whether and how the patterns of neural activity and behavior observed might arise from neural circuits, we explored how integration of neural activity could give rise to the waiting times. Although neural integration is proposed to be achieved by multiple neurons in a circuit ${ }^{2}$, here we modeled the integrator circuit as multiple input units feeding a single idealized integrator, similar to the drift-diffusion model.

In this section, $N\left(\mu, \sigma^{2}\right)$ denotes a Gaussian random variable with mean $\mu$ and variance $\sigma^{2}$. The model was run at discrete time step of $0.2 \mathrm{~s}$ until maximum time of $5 \mathrm{~s}$ ( 25 time steps) was reached.

Each input unit $i$ was assigned its own time of activation. At every time step, $t$, there were $N_{t}$ number of activated units $\left(N_{t}: 10,30,100\right.$ or 300). Each input unit was connected to the integrator with a random weight, $w_{i}$, drawn from $N\left(1 / N_{t}, 1 / N_{t}\right)$. To make sure the synaptic weights at each time step did not have strong bias toward positive or negative, we assigned a rank, $R_{w}$, to each input unit at a time step from 1 to $N_{t}$ and the synaptic weight was drawn randomly from a narrow percentile range (from $\left(R_{w}-1\right) / N_{t} \cdot 100$ to $R_{w} / N_{t} \cdot 100$ ) of the normal distribution, $N\left(1 / N_{t}, 1 / N_{t}\right)$. The activity of each unit, $F R_{i}(t)$ was described as a square pulse activation function as follows:

$$
\begin{gathered}
F R_{i}(t)=\mu_{F R}+\sigma_{F R} \cdot\left((1-\beta) \cdot N(0,1)+\beta \cdot z(w) \cdot N_{\mathrm{comm}}(t)\right), \text { at } t=T_{i} \\
F R_{i}(t)=0, \text { at } t \neq T_{i}
\end{gathered}
$$

where $\mu_{\mathrm{FR}}$ denotes mean firing rate with its value $10, \sigma_{F R}$ denotes trial-to-trial variability with its value $4, \beta$ denotes fraction of common noise varying from 0 to $0.6, z(w)$ denotes z-scored synaptic weight, $N_{\text {comm }}(t)$ denotes activity of the common noise source and $T_{i}$ denotes time of activation of each unit. $N_{\text {comm }}(t)$ was generated by convolving Gaussian white noise with Gaussian kernel with s.d. $0.6 \mathrm{~s}$ and then normalized to have 0 mean and unit variance. Negative $F R$ was always truncated at 0 .

Input units fed to a perfect integrator which kept adding to its own activity a weighted sum of all the input units' activity as follows:

$$
\begin{gathered}
F R_{\text {integ }}(t)=0, \quad \text { at } t=0 \\
F R_{\text {integ }}(t+1)=F R_{\text {integ }}(t)+\sum_{i=1}^{N} F R_{i}(t) \cdot w_{i}, \quad \text { at } t>0
\end{gathered}
$$

where $N_{\text {total }}=N_{t} \times 25$ (Note that only $N_{t}$ neurons were active at a particular time step). Once it reached a threshold level of 50 spikes per s, the model stops waiting. The model was run 100 times to produce 100 trials, comparable to the number of impatient trials used to analyze data neurons ( $101.7 \pm 1.8$ trials).

For the analysis in Figure 8b, correlation coefficient between the input units activity and the waiting time was examined.

For the trial-by-trial correlation analysis in Figure 8c, we analyzed 1,836 simultaneously recorded neuron pairs for data neurons (27 ramp-to-threshold neurons were excluded, because they were supposed to be the integrator neurons). For each neuron, the 0.4-s time bin with the lowest $P$ value for correlation between firing rates and waiting times was used for the analysis. Trial-by-trial partial correlations of firing rate of each pair of neurons were calculated factoring out 
correlation of firing rates with waiting times. Each pair of neurons was categorized as the same sign, opposite sign and others depending on the sign and significance of the firing rate correlation with waiting times. Each pair was also categorized by the absolute time difference of the most significant time bin (analysis time bin). For the model units, the activity at the 0.2 -s activated time was used to calculate the partial correlation.

\section{A Supplementary Methods Checklist is available.}

51. Holt, G.R., Softky, W. \& Koch, C. Comparison of discharge variability in vitro and in vivo in cat visual cortex neurons. J. Neurophysiol. 75, 1806-1814 (1996)

52. Paxinos, G. \& Watson, C. The Rat Brain in Stereotaxic Coordinates (Academic Press, 2005). 